\title{
Elastic fiber homeostasis requires lysyl oxidase-like 1 protein
}

\author{
Xiaoqing Liu ${ }^{1}$, Yun Zhao ${ }^{1}$, Jiangang Gao ${ }^{2}$, Basil Pawlyk ${ }^{1}$, Barry Starcher ${ }^{3}$, Jeffrey A Spencer ${ }^{4}$, Hiromi Yanagisawa ${ }^{4}$, \\ Jian $\mathrm{Zuo}^{2}$ \& Tiansen $\mathrm{Li}^{1}$
}

Elastic fibers are components of the extracellular matrix and confer resilience ${ }^{1}$. Once laid down, they are thought to remain stable $^{2}$, except in the uterine tract where cycles of active remodeling occur ${ }^{3}$. Loss of elastic fibers underlies connective tissue aging and important diseases including emphysema ${ }^{4-7}$. Failure to maintain elastic fibers is explained by a theory of antielastase-elastase imbalance ${ }^{8}$, but little is known about the role of renewal. Here we show that mice lacking the protein lysyl oxidase-like 1 (LOXL1) do not deposit normal elastic fibers in the uterine tract post partum and develop pelvic organ prolapse, enlarged airspaces of the lung, loose skin and vascular abnormalities with concomitant tropoelastin accumulation. Distinct from the prototypic lysyl oxidase (LOX), LOXL1 localizes specifically to sites of elastogenesis and interacts with fibulin-5. Thus elastin polymer deposition is a crucial aspect of elastic fiber maintenance and is dependent on LOXL1, which serves both as a cross-linking enzyme and an element of the scaffold to ensure spatially defined deposition of elastin.

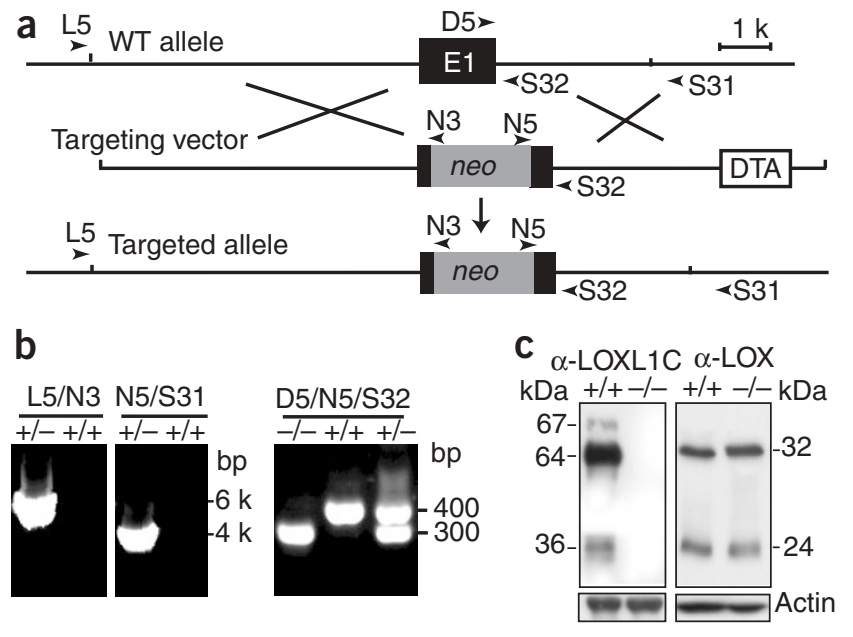

The main component of elastic fibers is an amorphous polymer composed of the protein elastin, known as tropoelastin in its monomeric form. Polymerization requires an initial step of oxidative deamination of lysine residues catalyzed by a lysyl oxidase ${ }^{9}$. The resulting aldehyde groups condense spontaneously with adjacent aldehydes or $\varepsilon$-amino groups of peptidyl lysine to form covalent cross-linkages. Lysyl oxidases are copper-dependent monoamine oxidases secreted by fibrogenic cells including fibroblasts and smooth muscle cells. Mammalian genomes have up to five potential LOX family members encoding the prototypic LOX and LOX-like proteins 1 through 4 (LOXL1, LOXL2, LOXL3 and LOXL4) $)^{9}$, but their individual roles in elastogenesis remain unclear. Elastogenesis also requires a scaffolding structure onto which elastin is deposited. Microfibrils, made up of fibrillins and microfibril-associated glycoproteins, are thought to serve as a scaffold that guides elastin deposition ${ }^{10}$. Inactivation of genes encoding fibrillin-1 and fibrillin-2 individually indicates that each may be dispensable for elastogenesis $^{11,12}$. Elastin binding protein (EBP) ${ }^{13}$, fibulin-5 (ref. 14) and an unspecified lysyl oxidase ${ }^{15}$ were also reported to associate with elastic

${ }^{1}$ Berman-Gund Laboratory for the Study of Retinal Degenerations, Harvard Medical School and Massachusetts Eye \& Ear Infirmary, Boston, Massachusetts 02114, USA. ${ }^{2}$ Department of Developmental Neurobiology, St. Jude Children's Research Hospital, Memphis, Tennessee 38105, USA. ${ }^{3}$ Department of Biochemistry, University of Texas Health Center at Tyler, Tyler, Texas 75708, USA. ${ }^{4}$ Department of Molecular Biology, University of Texas Southwestern Medical Center, Dallas, Texas 75390, USA. Correspondence should be addressed to T.L. (tli@meei.harvard.edu).

Published online 25 January 2004; doi:10.1038/ng1297 
Figure 2 Phenotype of mice lacking LOXL1 $\left(\right.$ Lox $\left./ 1^{--}\right)$. (a) Pelvic organ prolapse in Lox $/ 1^{-1-}$ female mice $2 \mathrm{~d}$ and $14 \mathrm{~d}$ post partum (PP2 and PP14). Although the prolapse retracted $14 \mathrm{~d}$ post partum, permanent pelvic descent remained, as indicated by a large genitourinary bulge. (b) The vaginal wall in Lox/1 ${ }^{-1-}$ females who had had multiple pregnancies was several times larger that in wild-type (WT) controls. The uterus was dilated and appeared translucent. (c) Airspace enlargement is visible in the mutant lung on gross inspection, particularly along the periphery. (d) Loose and redundant skin in the mutant. Mice shown in all panels were between 3 and 4 months of age. WT, wild-type.
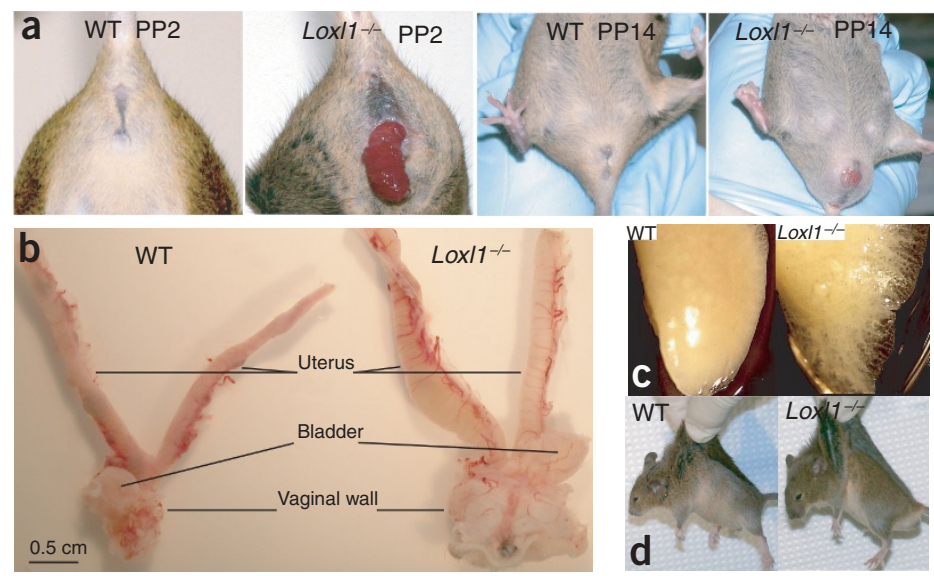

fibers. Gene targeting studies show that fibulin-5 is required for elastic fiber development ${ }^{14,16}$. Disruption of the gene Lox leads to a reduction in collagen and elastin cross-links and perinatal lethality ${ }^{17,18}$, suggestive of a role in cross-linking both fibrillar collagens and elastin during development.

To investigate a possible role for LOXL1 in elastogenesis, we ablated its expression in mice (Fig. 1). LOXL1-deficient mice were viable. Females were initially fertile but underwent pelvic prolapse $1-2 \mathrm{~d}$ post partum (Fig. 2a). Prolapsed tissues retracted over time but prominent pelvic descent remained, indicating permanent damage to the pelvic floor. Inspection of pelvic organs found the uterine cervix stretched to several times the normal size (Fig. 2b), consistent with a loss of resiliency. Loxl1 ${ }^{-/-}$mice of both genders developed enlarged airspaces of the lung (Fig. 2c), increased laxity and redundancy of the skin (Fig. 2d), rectal prolapse and intestinal diverticula (data not shown).

We found that LOXL1 was normally present in a stable fibrillar pattern that colocalized with elastic fibers (Fig. 3a) and was fully solubilized only under denaturing conditions (e.g., $6 \mathrm{M}$ urea). Elastic fibers in the uterine tract were thick, up to $3 \mu \mathrm{m}$ in diameter, and prominent under Nomarski optics (Fig. 3b). Uterine elastic fibers appeared normal in Loxl1 $1^{-/-}$females before pregnancy (data not shown). Pregnancy and birth, however, exposed the inability of the mutant to lay down normal elastic fibers during connective tissue remodeling. One week

Figure 3 Elastic fiber defect in Lox/1 $1^{-/-}$mice. (a) Immunofluorescence staining for elastin and LOXL1 in the post partum uterus (middle layer of myometrium is shown). In the wild-type (WT), elastin and LOXL1 colocalized in a fibrillar pattern. In the mutant, elastin staining was fragmented or aggregated. (b) Elastic fibers visualized under Nomarski optics and by immunostaining. Immunostaining for elastin (red) and LOXL1 (green) is shown only as an merged image (yellow). (c) Lung sections stained with hematoxylin and eosin show expanded alveoli in the mutant. (d) Loss of normal elastic fibers in the mutant skin (lower dermis) as shown by elastin immunostaining. (e) Elastic laminae of thoracic aorta shows weaker and diffuse immunostaining for elastin in the mutant. (f) Elastic laminae of thoracic aorta in the mutant has reduced amorphous elastin polymer shown by electron microscopy. (g) Immunoblots of total tissue homogenates detected by an elastin antibody. $72 \mathrm{kDa}$, elastin monomer (tropoelastin); $144 \mathrm{kDa}$, elastin dimer. Mice shown in all panels were between 3 and 5 months of age. Actin was used as a loading control. +/+, wild-type; -/-, homozygous Lox/1 $11^{-/}$mutant. (h) Quantification of desmosine and hydroxyproline. Values shown are means \pm s.d. Desmosine was reduced by $40-53 \%$ in the postpartum uterus (PPU), lung and skin, but not in the virgin uterus (VU) of the mutant. No significant differences were found in the hydroxyproline contents between the wild-type $(+/+)$ and mutant $(-/-)$ tissues. $n=5-10$ mice. ${ }^{*} P<0.05 ;{ }^{*} P<0.001$. WT, wild-type. post partum, Loxl1 ${ }^{-/-}$mice had fragmented elastin polymers as shown by immunostaining (Fig. 3a). Verhoeff and Hart's histochemical staining also indicated loss of elastic fibers. Electron microscopy showed collagen fibrils to be of comparable abundance and morphology in tissues of wild-type and Loxl1 ${ }^{-/-}$mice (data not shown).

Microscopic examinations found enlarged alveoli in the mutant lung (Fig. 3c), indicating emphysematous changes consistent with an
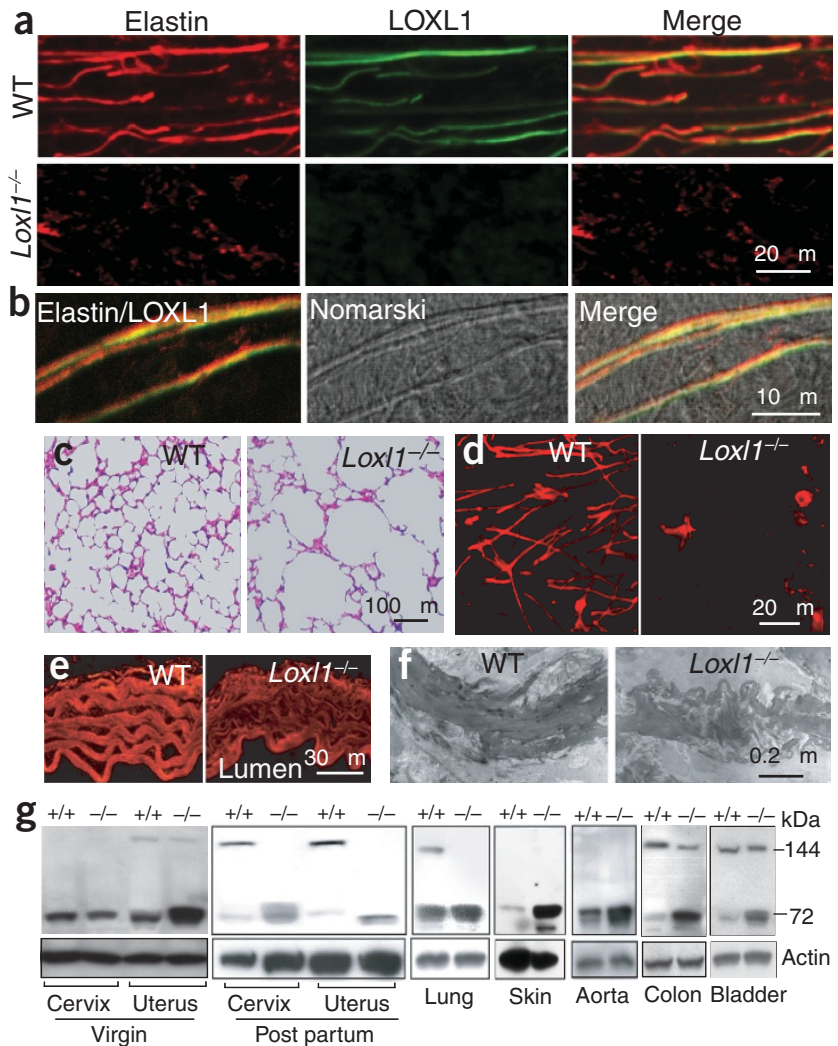

h
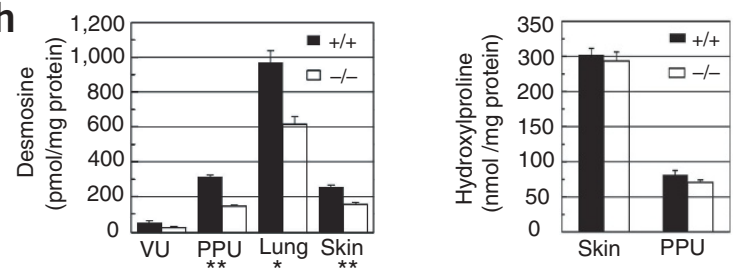
elastic fiber defect. Elastic fibers in the lower dermis of skin were also fragmented and reduced (Fig. 3d). Immunostaining for elastin in the elastic lamina of the aorta was diffuse and weak (Fig. 3e), suggestive of reduced and imprecise elastin polymer deposition. This suggestion was supported by ultrastructural examinations showing disorganized elastin core (Fig. 3f). Immunoblots showed that tropoelastin accumulated in multiple adult tissues, often with a concomitant reduction in cross-linked intermediate (Fig. $\mathbf{3 g}$ ). In older post partum uterine tissues, the elastin polymerization defect was much more pronounced than in young (30-d-old) virgin uterine tissues (Fig. 3g), indicating that the requirement for LOXL1 in the redeposition of elastin polymer increases through the reproductive cycle. These data also indicate that new elastin polymer deposition may continue in wild-type adult tissues but is arrested by the loss of LOXL1. A selective role for LOXL1 in elastin but not collagen metabolism is supported by measurements of desmosine and hydroxyproline (Fig. $3 \mathbf{h}$ ), which represent elastin and collagen cross-links, respectively. Desmosine levels were significantly lower in multiple Loxl1 $^{-/-}$tissues than in corresponding wild-type tissues, whereas hydroxyproline levels remained unchanged.

LOXL1 is closely related to the prototypic LOX, and both are widely expressed. Although no difference in substrate selectivity was detected
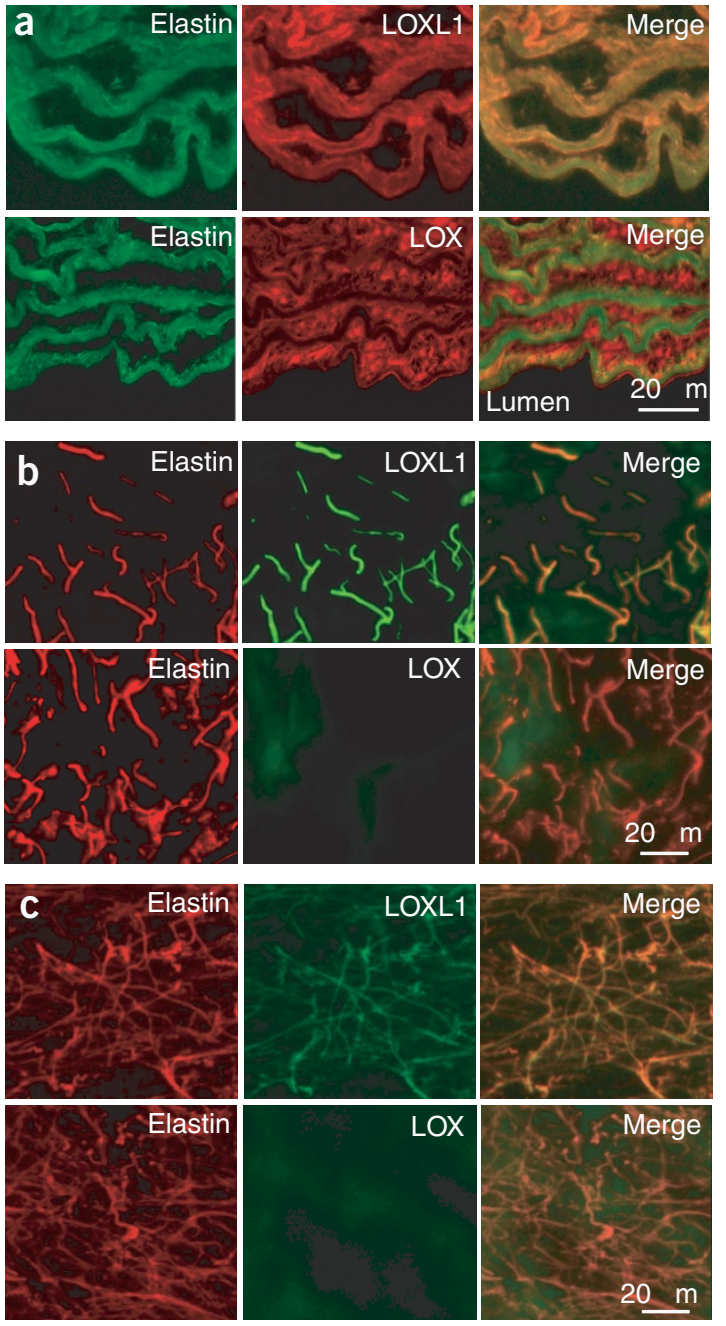

Figure 4 Differential localization of LOXL1 and LOX in relation to elastic fibers. Double labeling for elastin and LOXL1 or LOX in cross-sections of the aorta (a), lower dermis of skin (b) and myometrium layer of uterus (c). in vitro, the inability of LOX to compensate for LOXL1 and the largely nonoverlapping phenotypes of the gene-ablated mutants ${ }^{17,18}$ are suggestive of functional differences in vivo. By immunolocalization using monospecific antibodies, LOXL1 was closely associated with the elastic lamina whereas LOX was broadly distributed (Fig. 4a). In the skin (lower dermis; Fig. 4b) and the uterus (myometrium; Fig. 4c), we found LOXL1 fully overlapping with elastic fibers, whereas LOX was diffusely distributed. These data indicate that LOXL1, but not LOX, is specifically targeted to sites of elastogenesis. Thus, LOXL1 seems to function primarily to guide elastin deposition in a spatially defined manner, a prerequisite for the formation of functional elastic fibers. The localization pattern of LOX, on the other hand, seems consistent with a role in cross-linking both collagens and elastin ${ }^{17,18}$.

To determine the molecular interactions that could account for the targeting of LOXL1 to the scaffold of elastogenesis, we carried out protein interaction screens using the yeast two-hybrid system. With LOXL1 as a bait, we found fibulin- 5 to be a potential interacting partner. Further analyses showed that a region immediately $\mathrm{N}$-terminal to the catalytic domain (L3) of LOXL1 interacted with the Cterminal portion (F3) of fibulin-5 (Fig. 5a). Their physical interaction in vitro was confirmed using pull-down assays (Fig. 5b). Coimmunoprecipitation of fibulin-5 with LOXL1 from tissue extracts (Fig. 5c) indicated an in vivo interaction. In cell culture, LOXL1 and fibulin-5 colocalized in a perinuclear compartment in transiently transfected COS-7 cells (data not shown) and in extracellular deposits produced by vascular smooth muscle cells (Fig. $\mathbf{5 d}$ ). In tissue sections, LOXL1 and fibulin-5 colocalized in a fibrillar pattern that also fully overlapped with elastin immunostaining (Fig. 5e). Loss of fibulin-5 abolished the fibrillar staining pattern of LOXL1, but not vice versa (Fig. 5e). These observations indicate that fibulin-5 may localize to sites of elastogenesis independent of LOXL1 and that fibulin-5 might be responsible for tethering LOXL1 to these sites. LOXL1, on the other hand, seems to stabilize the fibrillar form of fibulin- 5 as indicated by the increased solubility of fibulin- 5 in absence of LOXL1 (Fig. 5f). The increased solubility of fibulin-5 may be due to disruption of elastin polymer in the LOXL1 mutant tissues. Loss of LOXL1 also increased the soluble fraction of EBP, a protein known to associate with elastic fibers. Fibrillin-1 and fibrillin-2, components of microfibrils, were only partially affected by the loss of LOXL1 (Fig. 5f), consistent with the fact that not all microfibrils are associated with elastic fibers.

Further support comes from the similarity between the two mutant mouse models, both of which show specific defects in elastogenesis. In general, elastic fiber defect manifests earlier in the fibulin-5 mutant than in the LOXL1 mutant. For example, a $1 \mathrm{~d}$-old fibulin-5 mutant showed tortuosity of the aorta, which was not seen in an age-matched LOXL1 mutant (data not shown). Young ( $30 \mathrm{~d}$-old) LOXL1 mutant mice had abundant elastic fibers in their uterine tract and skin, as shown by elastin immunostaining, whereas young fibulin-5 mutant mice had few elastic fibers in these tissues. Although a role for LOXL1 in elastic fiber development could not be ruled out, these phenotypic differences are consistent with the idea that the elastic fiber defect seen in the LOXL1 mutant can be attributed, at least in part, to a defect in elastic fiber renewal in adult tissues.

We present a model to illustrate how LOXL1 might function during elastic fiber renewal (Fig. 5g). Binding of fibulin-5 to both tropoelastin ${ }^{14}$ and LOXL1 brings the enzyme and substrate into juxtaposition for efficient and spatially restricted polymer formation. LOXL1 converts tropoelastin into a lysyl-deaminated form, and the 'activated' tropoelastin associates with one another or deposits onto the existing polymer through coacervation ${ }^{19}$, followed by spontaneous covalent cross-linking. This allows addition of elastin polymer 
at the interface next to the scaffold. LOXL1 binding to fibulin-5 may also have a regulatory role, given that cleavage of the $\mathrm{N}$-terminal portion of LOXL1 activates its activity in vitro ${ }^{20}$, yet LOXL1 is present predominantly in the full-length form in vivo.

This study shows that LOXL1 has a generalized, nonredundant role in elastic fiber homeostasis in adult tissues. We speculate that genetic defects in human LOXL1 may lead to clinical syndromes resembling generalized elastolysis affecting skin, lung, large arteries and other organs (e.g., cutis laxa type I; OMIM 219100). Our data also show an unexpected, causal link between elastic fiber homeostasis and pelvic prolapse, a clinical condition strongly correlated with both childbirth and advanced age $\mathrm{e}^{21-23}$. Elastin polymer and soluble elastin-derived peptides also have signaling roles in cell adhesion, migration and proliferation ${ }^{5,24}$. Thus, the combination of loss of elastin polymer and accumulation of tropoelastin may have additionally contributed to the histopathologic changes seen in the LOXL1 mutant mice.

Diseases characterized by loss of elastic fibers, such as emphysema, are usually viewed in terms of an imbalance between elastases and their inhibitors ${ }^{8,25,26}$. Given our finding that LOXL1-dependent elastin polymer deposition has an active role in elastic fiber homeostasis, this theory might be updated to include an imbalance between degradation and renewal as well. According to this view,
LOXL1 could be a second target, after elastases, affected by factors that promote elastic fiber loss.

\section{METHODS}

Gene targeting. We amplified genomic fragments flanking exon 1 of the Loxl1 gene from $129 / \mathrm{Sv}$ mouse DNA by PCR. We cloned these fragments into the pGT-N29 vector on either side of the neomycin-resistance gene. The targeting vector also contained a diphtheria toxin expression cassette as a negative-selection marker (Fig. 1a). We linearized the targeting vector and electroporated it into R1 embryonic stem (ES) cells. We identified multiple targeted ES clones by PCR (Fig. 1b), microinjected targeted clones into C57BL/6 blastocysts and crossed the chimeras with C57BL/6 mice. We identified mice heterozygous or homozygous with respect to the targeted allele by PCR (Fig. 1b). Primer sequences are available on request. Homozygous fibulin-5-mutant mice have been described ${ }^{14}$. The animal care and use committee of the Massachusetts Eye \& Ear Infirmary approved all animal protocols.

Antibodies, immunoblotting and immunofluorescence. We generated two polyclonal antibodies to LOXL1 and one antibody to LOX by immunizing rabbits with His-tagged recombinant proteins (sequences in GenBank; see accession numbers below). Antibody to LOXL1N (spanning amino acid residues 175-313 of mouse LOXL1) is unique to LOXL1 but does not recognize the cleavage product ( $36 \mathrm{kDa}$; Fig. 1c). Antibodies to LOXL1C (spanning residues 313-607) and to LOX (spanning residues 204-411 of mouse LOX)


Figure 5 In vitro and in vivo interaction between LOXL1 and fibulin-5. (a) Protein interaction screen by yeast two-hybrid analyses. The maximal interaction domains reside in regions L3 and F3. S, secretory signal sequence. (b) GST pull-down assay showing in vitro direct interaction between fibulin-5 and LOXL1. Input, $16 \%$ of eluate. (c) Coimmunoprecipitation of fibulin-5 with LOXL1 in lung extracts, suggestive of interaction in vivo. Input, $50 \%$ of eluate. (d) Colocalization of LOXL1 and fibulin-5 in extracellular matrix deposited by a cultured vascular smooth muscle cell line (PAC-1). The two proteins are found together in fibrillar and dot patterns. (e) Double labeling for elastin and fibulin-5 or for LOXL1 and fibulin-5 in wild-type (WT) and mutant mouse tissue sections. Fbln $5^{-1-}$, fibulin-5 mutant. Sections from lower dermis are shown, but results from uterine tissues are comparable. (f) Immunoblot analyses of elastic fiber-associated proteins from wild-type $(+/+)$ and $L o x / 1^{-/-}(-/-)$uterine tissues. PBS, tissue extracts with PBS; urea, tissue extracts with 6 M urea after PBS extraction. Fibulin-5 and EBP in the mutant were mostly shifted to the soluble fraction (PBS extractable) from an insoluble fraction (urea extractable). In the rightmost panel, a band of $67 \mathrm{kDa}$ (arrowhead) represents EBP. The identity of a smaller (59-kDa) protein is uncertain, but it was previously found in uterus and may be a smaller EBP isoform ${ }^{30}$. (g) A simplified model for the role of LOXL1 in elastogenesis. Possible tissue-specific variations and partial functional redundancies are not considered. The nature of the interaction between fibulin- 5 and microfibrils remains unclear. $\mathrm{TE}$, tropoelastin. The $\mathrm{N}$-terminal unique region of LOXL1 binds to fibulin-5. C, C-terminal domains. 
encompassed the C-terminal region conserved among LOX family members. To obtain antibodies that were monospecific, we put affinity-purified antibodies to LOXL1C and to LOX through columns containing the respective homologous proteins immobilized on agarose beads (AminoLink Plus, Pierce). Fibulin-5 antibody (BSYN1923) made in rabbits was described previously ${ }^{14}$. The following antibodies to elastin, fibrillin-1 and fibrillin-2 were from Elastin Products Company: PR385 (rabbit antibody to mouse elastin, exons 6-17); PR387 (rabbit antibody to mouse elastin, exons 31-36); RT675 (goat antibody to rat elastin) used for double labeling of elastin with LOXL1, LOX or fibulin-5; PR210AP (rabbit antibody to fibrillin-1); PR225 (rabbit antibody to fibrillin-2). EBP antibody (EBP2112) $)^{5}$ was a gift from A. Hinek (The Hospital for Sick Children, Toronto, Canada). We carried out immunoblotting and immunofluorescence staining of unfixed cryosections as described ${ }^{27}$. Secondary antibodies conjugated to Alexa 488 (green) and Alexa 594 (red) were from Molecular Probes. To obtain double labeling for LOXL1 and fibulin-5, we tagged LOXL1 antibody with biotin using the MiniBiotin protein labeling kit (Molecular Probes) and detected it with fluorochrome-conjugated streptavidin. For immunoblotting, we used total tissue homogenates to detect soluble elastin (monomer and dimer) probed with antibody PR385. Unless otherwise noted, we extracted tissues first with phosphate-buffered saline (PBS) and then with $6 \mathrm{M}$ urea, and used the urea extracts for immunoblotting analysis of LOXL1 and LOX.

Yeast two-hybrid screens. We used the Matchmaker system 3 (Clontech) for yeast two-hybrid screens as previously described ${ }^{27}$. An adult rat lung library in the pACT2 vector was from Clontech. The initial library screen used the full-length LOXL1 as the bait. To determine the interacting domains, we prepared a series of deletion constructs (Fig. 5a) and tested them by cotransformation in yeast.

Cell cultures. We inserted full-length cDNAs encoding fibulin-5 and LOXL1 (without the signal peptide sequences) into pcDNA3 and the pEGFP-C2 vectors, respectively. After transient transfection in COS-7 cells, recombinant proteins were visualized with the aid of the EGFP tag and by staining with the fibulin-5 antibody. To examine colocalization of LOXL1 and fibulin-5 in the extracellular matrix, we cultured a rat arterial smooth muscle cell line (PAC-1) known to deposit extracellular matrix material ${ }^{28}$ and carried out double-labeling immunofluorescence for LOXL1 and fibulin-5.

Light and transmission electron microscopy. We fixed mouse tissues in $4 \%$ formaldehyde and embedded them in paraffin. The trachea and lungs were removed together and fixed under $20 \mathrm{~cm}$ water pressure. We carried out histochemical (Verhoeff and Hart's stain) staining and histologic examinations of multiple tissues on paraffin-embedded sections. For electron microscopy, we fixed tissues in $2.5 \%$ glutaraldehyde, $1 \%$ formaldehyde in sodium cacodylate buffer and embedded them in Epon.

Glutathione S-transferase (GST) pull-downs and coimmunoprecipitation. We inserted the L3 and F3 coding sequences (Fig. 5a) into pET-28a and pGEX$4 \mathrm{~T} 1$ vectors, respectively, and used purified soluble fractions for the GST pulldown assay. We used Glutathione Sepharose 4 Fast Flow beads (Pharmacia) to pull down GST and GST-L3, and probed inputs and eluates with antibody to T7 Tag conjugated to horseradish peroxidase (Novagen) and chicken antibodies to GST on immunoblots. For coimmunoprecipitation, we extracted wildtype mouse lungs with $4 \mathrm{M}$ urea, dialyzed the supernatant overnight at $4{ }^{\circ} \mathrm{C}$ against PBS and carried out immunoprecipitation with antibody to LOXL1C and protein $\mathrm{G}-$ agarose.

Desmosine and hydroxyproline analysis. We carried out these assays as described ${ }^{29}$.

GenBank accession numbers. LOXL1 protein, AAK97375; LOX protein, AAH18439.

\section{ACKNOWLEDGMENTS}

We thank M. Adamian and N. Michaud for histologic preparations and J. Yang, O. Bulgakov and M. Sandberg for helpful advice. This work was supported by the
Ruth and Milton Steinbach Fund (T.L.) and by grants from the National Institutes of Health (J.Z. and H.Y.) and the American Heart Association (H.Y.).

\section{COMPETING INTERESTS STATEMENT}

The authors declare that they have no competing financial interests.

Received 23 September; accepted 29 December 2003

Published online at http://www.nature.com/naturegenetics

1. Mecham, R.P. \& Davis, E. Elastic fiber structure and assembly. in Extracel/ular Matrix Assembly and Structure (eds. Yurchenco, P.D., Birk, D.E. \& Mecham, R.P.) 281-314 (Academic, New York, 1994)

2. Davis, E.C. Stability of elastin in the developing mouse aorta: a quantitative radioautographic study. Histochemistry 100, 17-26 (1993).

3. Starcher, B. \& Percival, S. Elastin turnover in the rat uterus. Connect. Tissue. Res. 13, 207-215 (1985)

4. Blumenkranz, M.S., Russell, S.R., Robey, M.G., Kott-Blumenkranz, R. \& Penneys, N. Risk factors in age-related maculopathy complicated by choroidal neovascularization. Ophthalmology 93, 552-558 (1986).

5. Mochizuki, S., Brassart, B. \& Hinek, A. Signaling pathways transduced through the elastin receptor facilitate proliferation of arterial smooth muscle cells. J. Biol. Chem. 277, 44854-44863 (2002).

6. Hasham, S.N., Guo, D.C. \& Milewicz, D.M. Genetic basis of thoracic aortic aneurysms and dissections. Curr. Opin. Cardiol. 17, 677-683 (2002).

7. Pasquali-Ronchetti, I. \& Baccarani-Contri, M. Elastic fiber during development and aging. Microsc. Res. Tech. 38, 428-435 (1997).

8. Shapiro, S.D. The pathogenesis of emphysema: the elastase:antielastase hypothesis 30 years later. Proc. Assoc. Am. Physicians 107, 346-352 (1995).

9. Kagan, H.M. \& Li, W. Lysyl oxidase: properties, specificity, and biological roles inside and outside of the cell. J. Cell. Biochem. 88, 660-672 (2003).

10. Kielty, C.M., Sherratt, M.J. \& Shuttleworth, C.A. Elastic fibres. J. Cell. Sci. 115, 2817-2828 (2002)

11. Pereira, L. et al. Targeting of the gene encoding fibrillin-1 recapitulates the vascular aspect of Marfan syndrome. Nat. Genet. 17, 218-222 (1997).

12. Chaudhry, S.S. et al. Mutation of the gene encoding fibrillin-2 results in syndactyly in mice. Hum. Mol. Genet. 10, 835-843 (2001).

13. Hinek, A. \& Rabinovitch, M. 67-kD elastin-binding protein is a protective "companion" of extracellular insoluble elastin and intracellular tropoelastin. J. Cell. Biol. 126, 563-574 (1994)

14. Yanagisawa, H. et al. Fibulin-5 is an elastin-binding protein essential for elastic fibre development in vivo. Nature 415, 168-171 (2002).

15. Kagan, H.M., Vaccaro, C.A., Bronson, R.E., Tang, S.S. \& Brody, J.S. Ultrastructural immunolocalization of lysyl oxidase in vascular connective tissue. J. Cell. Biol. 103, $1121-1128$ (1986)

16. Nakamura, T. et al. Fibulin-5/DANCE is essential for elastogenesis in vivo. Nature 415, 171-175 (2002)

17. Maki, J.M. et al. Inactivation of the lysyl oxidase gene Lox leads to aortic aneurysms, cardiovascular dysfunction, and perinatal death in mice. Circulation 106, 2503-2509 (2002).

18. Hornstra, I.K. et al. Lysyl oxidase is required for vascular and diaphragmatic development in mice. J. Biol. Chem. 278, 14387-14393 (2003)

19. Vrhovski, B. \& Weiss, A.S. Biochemistry of tropoelastin. Eur. J. Biochem. 258, 1-18 (1998).

20. Borel, A. et al. Lysyl oxidase-like protein from bovine aorta. Isolation and maturation to an active form by bone morphogenetic protein-1. J. Biol. Chem. 276, 48944-48949 (2001)

21. Koduri, S. \& Sand, P.K. Recent developments in pelvic organ prolapse. Curr. Opin. Obstet. Gynecol. 12, 399-404 (2000).

22. Sultan, A.H., Monga, A.K. \& Stanton, S.L. The pelvic floor sequelae of childbirth. Br. J. Hosp. Med. 55, 575-579 (1996).

23. Snooks, S.J., Swash, M., Mathers, S.E. \& Henry, M.M. Effect of vaginal delivery on the pelvic floor: a 5-year follow-up. Br. J. Surg. 77, 1358-1360 (1990).

24. Karnik, S.K. et al. A critical role for elastin signaling in vascular morphogenesis and disease. Development 130, 411-423 (2003).

25. Hautamaki, R.D., Kobayashi, D.K., Senior, R.M. \& Shapiro, S.D. Requirement for macrophage elastase for cigarette smoke-induced emphysema in mice. Science $\mathbf{2 7 7}$ 2002-2004 (1997).

26. Morris, D.G. et al. Loss of integrin $\alpha v \beta 6$-mediated TGF- $\beta$ activation causes Mmp12dependent emphysema. Nature 422, 169-173 (2003).

27. Hong, D.H., Yue, G., Adamian, M. \& Li, T. Retinitis pigmentosa GTPase regulator (RPGR)-interacting protein is stably associated with the photoreceptor ciliary axoneme and anchors RPGR to the connecting cilium. J. Biol. Chem. 276, 12091-12099 (2001).

28. Rothman, A. et al. Development and characterization of a cloned rat pulmonary arterial smooth muscle cell line that maintains differentiated properties through multiple subcultures. Circulation 86, 1977-1986 (1992).

29. Starcher, B. \& Conrad, M. A role for neutrophil elastase in solar elastosis. Connect. Tissue Res. 31, 133-140 (1995).

30. Yamamoto, M., Akazawa, K., Aoyagi, M., Yamamoto, N. \& Yamamoto, K. Changes in elastin-binding protein in fibroblasts derived from cardinal ligaments of patients with prolapsus uteri. Cell Biol. Int. 26, 441-449 (2002). 